TRANSACTIONS OF THE

AMERICAN MATHEMATICAL SOCIETY

Volume 362, Number 10, October 2010, Pages 5333-5353

S 0002-9947(2010)04891-3

Article electronically published on May 20, 2010

\title{
GAUSSIAN BRUNN-MINKOWSKI INEQUALITIES
}

\author{
RICHARD J. GARDNER AND ARTEM ZVAVITCH
}

\begin{abstract}
A detailed investigation is undertaken into Brunn-Minkowski-type inequalities for Gauss measure. A Gaussian dual Brunn-Minkowski inequality, the first of its type, is proved, together with precise equality conditions, and is shown to be the best possible from several points of view. A new Gaussian Brunn-Minkowski inequality is proposed and proved to be true in some significant special cases. Throughout the study attention is paid to precise equality conditions and conditions on the coefficients of dilatation. Interesting links are found to the S-inequality and the (B) conjecture. An example is given to show that convexity is needed in the (B) conjecture.
\end{abstract}

\section{INTRODUCTION}

This paper focuses on two fundamental ingredients of mathematics: Gauss measure, the most important probability measure in $\mathbb{R}^{n}$, and the Brunn-Minkowski inequality, one of the most powerful inequalities in analysis and geometry.

The Brunn-Minkowski inequality for convex bodies $K$ and $L$ in $\mathbb{R}^{n}$ states that

$$
V_{n}(K+L)^{1 / n} \geq V_{n}(K)^{1 / n}+V_{n}(L)^{1 / n},
$$

where $K+L$ is the Minkowski or vector sum of $K$ and $L, V_{n}$ denotes $n$-dimensional Lebesgue measure, and equality holds if and only if $K$ is homothetic to $L$. (See Section 2 for unexplained notation and terminology.) By the homogeneity of $V_{n}$, this is equivalent to

$$
V_{n}(s K+t L)^{1 / n} \geq s V_{n}(K)^{1 / n}+t V_{n}(L)^{1 / n},
$$

where $s, t \geq 0$.

It is known that (1) and (2) still hold when the sets concerned are Lebesgue measurable, and indeed the Brunn-Minkowski inequality reaches far beyond geometry. No less than three recent surveys cover its extensive generalizations, variations, connections, and applications in probability and statistics, information theory, Banach space theory, algebraic geometry, geometric tomography, interacting gases, and crystallography; see [1, [14, and [28].

The Brunn-Minkowski inequality (11) is a cornerstone of the vast Brunn-Minkowski theory, expounded in 29]. This harbors the tools, such as Minkowksi sum, for metrical problems on convex bodies and their projections onto subspaces. Around

Received by the editors November 13, 2007 and, in revised form, July 3, 2008.

2010 Mathematics Subject Classification. Primary 52A20, 52A40, 60E15, 60G15.

Key words and phrases. Convex body, star body, geometric tomography, Gauss measure, Brunn-Minkowski inequality, Ehrhard's inequality, dual Brunn-Minkowski theory, radial sum.

This work was supported in part by U.S. National Science Foundation grants DMS-0603307 and DMS-0504049.

(C)2010 American Mathematical Society Reverts to public domain 28 years from publication 
1975, Lutwak 27] observed that when the Minkowski sum of two sets is replaced by an operation he called radial sum, in which only sums of parallel vectors are taken into account, a theory arises that is ideal for treating metrical problems about sets star-shaped with respect to the origin, and their intersections with subspaces. This newer theory, now called the dual Brunn-Minkowski theory, has attracted much attention and counts among its successes the solution of the 1956 Busemann-Petty problem on volumes of central sections of $o$-symmetric convex bodies; see [15], [16], [17, 26], 30, and 31.

Corresponding in the dual theory to the Brunn-Minkowski inequality (1) is the dual Brunn-Minkowski inequality for bounded Borel star sets $C$ and $D$ in $\mathbb{R}^{n}$, which states that

$$
V_{n}(C \tilde{+} D)^{1 / n} \leq V_{n}(C)^{1 / n}+V_{n}(D)^{1 / n}
$$

where $\widetilde{+}$ denotes radial sum, with equality if and only if $C$ is a dilatate of $D$. See, for example, [13, (B.30)] and [18, Section 3]. This is equivalent to

$$
V_{n}(s C \tilde{+} t D)^{1 / n} \leq s V_{n}(C)^{1 / n}+t V_{n}(D)^{1 / n},
$$

where $s, t \geq 0$. The reversal of the inequality sign in the passage from (11) to (3) is a standard, but not yet fully understood, feature of the duality at play.

Here we are interested in inequalities of the Brunn-Minkowski type for Gauss measure $\gamma_{n}$ in $\mathbb{R}^{n}$. Despite the fact that $\gamma_{n}$ is not translation invariant, such inequalities have been found and in fact have generated substantial literature. The most powerful, due to Ehrhard [1], 12, states that for $0<t<1$ and closed convex sets $K$ and $L$ in $\mathbb{R}^{n}$, we have

$$
\Phi^{-1}\left(\gamma_{n}((1-t) K+t L)\right) \geq(1-t) \Phi^{-1}\left(\gamma_{n}(K)\right)+t \Phi^{-1}\left(\gamma_{n}(L)\right),
$$

where $\Phi(x)=\gamma_{1}((-\infty, x))$. By [12, p. 154], equality holds when $\gamma_{n}(K) \gamma_{n}(L)>0$ if and only if $K=\mathbb{R}^{n}, L=\mathbb{R}^{n}, K=L$, or both $K$ and $L$ are half-spaces, one contained in the other. Since the function $\Phi$ is (strictly) $\log$ concave (i.e., $\log \Phi$ is (strictly) concave), Ehrhard's inequality and its equality condition imply that for $0<t<1$ and closed convex sets $K$ and $L$ in $\mathbb{R}^{n}$,

$$
\gamma_{n}((1-t) K+t L) \geq \gamma_{n}(K)^{1-t} \gamma_{n}(L)^{t}
$$

with equality when $\gamma_{n}(K) \gamma_{n}(L)>0$ if and only if $K=L$. Inequality (6), proved independently by Borell [3], [4] and Brascamp and Lieb [9], is also an easy consequence of the Prékopa-Leindler inequality and the fact that the density function of $\gamma_{n}$ is $\log$ concave, and moreover (6) holds when the sets concerned are Borel sets (see, for example, [14, p. 378]). On the other hand it was only recently that Borell 6] proved that (5) also holds for Borel sets. (Note that what Borell in [5] calls the Brunn-Minkowski inequality for Gauss measure is none of the above inequalities but is rather an isoperimetric inequality that follows from (5]); see [22].)

One of our main results, and the original motivation for the paper, is the following new inequality for Borel star sets $C$ and $D$ in $\mathbb{R}^{n}$ and $s, t \geq 1$ :

$$
\gamma_{n}(s C \tilde{+} t D)^{1 / n} \leq s \gamma_{n}(C)^{1 / n}+t \gamma_{n}(D)^{1 / n} .
$$

See Theorem 4.2, which also gives precise equality conditions. What is remarkable about this Gaussian dual Brunn-Minkowski inequality (compare (44)) is not its proof, which does not require innovative techniques, but that it exists. The discussion after Theorem 4.2 shows that the inequality is the best possible from several points 
of view. In particular, the restriction $s, t \geq 1$ on the coefficients of dilatation is necessary. This may seem strange at first, since (4) has no such restriction. However, $\gamma_{n}$ is not homogeneous, and the restriction $s, t \geq 1$ becomes natural when we see that it also applies to (4) when the exponent $1 / n$ is replaced by $0<p<1 / n$. See Section 3 , where we examine the role of the coefficients of dilatation in several inequalities, including, for the first time as far as we know, those for (6).

Also in Section 3 we find that when the exponent $1 / n$ in (4) is replaced by $p>1 / n$, the appropriate condition on the coefficients is $s+t \leq 1$, which includes the important special case of the convex combination where $s=1-t$. This raises the question (see Question 6.1) as to whether there is a Gaussian dual BrunnMinkowski inequality that holds when $s+t \leq 1$. Our investigation in Section 6 turns up an interesting connection with the so-called S-inequality of Latała and Oleszkiewicz [24], but our results suggest that there may be no satisfactory answer to this question.

In the course of our detailed investigation into Gaussian dual Brunn-Minkowski inequalities, we were led to the following intriguing question (see Question [7.1): If $0<t<1$ and $K$ and $L$ are closed convex sets containing the origin in $\mathbb{R}^{n}$, is it true that

$$
\gamma_{n}((1-t) K+t L)^{1 / n} \geq(1-t) \gamma_{n}(K)^{1 / n}+t \gamma_{n}(L)^{1 / n} ?
$$

In Section [7, we note that the restriction on the position of $K$ and $L$ is necessary, but in view of the direct analogy with (2), it is amazing that the inequality seems to have been overlooked. It does not follow from Ehrhard's inequality (5), and if true it would be stronger than (6) when $K$ and $L$ contain the origin. We provide evidence in its favor by showing that it is true when $K$ and $L$ are coordinate boxes, when either $K$ or $L$ is a slab, and when $K$ and $L$ are both dilatates of the same $o$-symmetric closed convex set. Even the latter special case is not at all easy. We establish it by means of a fascinating link (see Theorem 17.6) with Banaszczyk's conjecturethe (B) conjecture - that $\gamma_{n}\left(e^{t} K_{0}\right)$ is log concave in $t$ when $K_{0}$ is an $o$-symmetric convex body, recently proved by Cordero-Erasquin, Fradelizi, and Maurey [10]. It is not known if the symmetry is necessary for the truth of the $(\mathrm{B})$ conjecture, but we give an example to show that the convexity is necessary. In Theorem 8.1 we prove a Gaussian Prékopa-Leindler inequality that follows from earlier results.

We are very grateful to Franck Barthe for his helpful suggestions and comments, in particular the contribution given in detail at the end of Section 7

\section{Definitions, notation, And PRELiminaries}

As usual, $S^{n-1}$ denotes the unit sphere, $B$ the unit ball, $o$ the origin, and $\|\cdot\|$ the norm in Euclidean $n$-space $\mathbb{R}^{n}$. If $x, y \in \mathbb{R}^{n}$, then $x \cdot y$ is the inner product of $x$ and $y$ and $[x, y]$ denotes the line segment with endpoints $x$ and $y$.

If $X$ is a set, $\operatorname{dim} X$ is its dimension, that is, the dimension of its affine hull, and $\partial X$ is its boundary. A set is o-symmetric if it is centrally symmetric, with center at the origin. If $r \in \mathbb{R}$, the set $r X=\{r x: x \in X\}$ is called a dilatate of $X$. If $X$ and $Y$ are sets in $\mathbb{R}^{n}$, then

$$
X+Y=\{x+y: x \in X, y \in Y\}
$$

is the Minkowski or vector sum of $X$ and $Y$.

A body is a compact set equal to the closure of its interior. 
We write $V_{k}$ for $k$-dimensional Lebesgue measure in $\mathbb{R}^{n}$, where $k=1, \ldots, n$ and where we identify $V_{k}$ with $k$-dimensional Hausdorff measure. If $K$ is a $k$-dimensional body in $\mathbb{R}^{n}$, then we refer to $V_{k}(K)$ as its volume. Define $\kappa_{n}=V_{n}(B)$. The notation $d z$ will always mean $d V_{k}(z)$ for the appropriate $k=1, \ldots, n$.

A set in $\mathbb{R}^{n}$ is called a convex body if it is convex and compact with nonempty interior. The treatise of Schneider 29] is an excellent general reference for convex sets.

A (possibly unbounded) set $C$ is star shaped at the origin if every line through the origin that meets $C$ does so in a (possibly degenerate) closed line segment, a closed half-infinite ray, or in the line itself. If $C$ is a set that is star shaped at the origin, its radial function $\rho_{C}$ is defined, for all $u \in S^{n-1}$ such that the line through the origin parallel to $u$ intersects $C$, by

$$
\rho_{C}(u)=\sup \{c \in \mathbb{R}: c u \in C\} .
$$

Note that $C$ may not contain the origin and that $\rho_{C}$ may take negative or infinite values. In this paper, a Borel star set is a Borel set that contains the origin and is star shaped at the origin.

By a star body in $\mathbb{R}^{n}$ we mean a body $L$ star shaped at the origin such that $\rho_{L}$, restricted to its support, is continuous. This definition, introduced in [19] (see also [13, Section 0.7]), allows bodies not containing the origin, unlike previous definitions; in particular, every convex body is a star body in this sense.

If $x, y \in \mathbb{R}^{n}$, then the radial sum $x \widetilde{+} y$ of $x$ and $y$ is defined to be the usual vector sum $x+y$ if $x$ and $y$ are contained in a line through $o$, and $o$ otherwise. If $C$ and $D$ are Borel star sets in $\mathbb{R}^{n}$ and $s, t \in \mathbb{R}$, then

$$
s C \tilde{+} t D=\{s x \tilde{+} t y: x \in C, y \in D\}
$$

and

$$
\rho_{s C \tilde{+} t D}=s \rho_{C}+t \rho_{D} .
$$

The standard Gauss measure $\gamma_{n}$ is defined for measurable subsets $E$ of $\mathbb{R}^{n}$ by

$$
\gamma_{n}(E)=c_{n} \int_{E} e^{-\|x\|^{2} / 2} d x
$$

where $d x$ denotes integration with respect to $V_{n}$ and

$$
c_{n}=(2 \pi)^{-n / 2} \text {. }
$$

For $n \in \mathbb{N}$ and $r \in \mathbb{R}$, define

$$
\Psi_{n}(r)=\gamma_{n}(r B) .
$$

From (8) it follows by substitution that if $E$ is a measurable subset of $\mathbb{R}^{n}$, then

$$
\gamma_{n}(s E)^{1 / n} \geq s \gamma_{n}(E)^{1 / n} \text { if } 0 \leq s \leq 1 \text { and } \gamma_{n}(s E)^{1 / n} \leq s \gamma_{n}(E)^{1 / n} \text { if } s \geq 1 \text {. }
$$

Equality holds in each inequality if and only if $s=1$ or $\gamma_{n}(E)=0$.

Let

$$
\Phi(x)=\frac{1}{\sqrt{2 \pi}} \int_{-\infty}^{x} e^{-t^{2} / 2} d t
$$

and note that $\Phi(x)=\gamma_{1}((-\infty, x))$.

It will be convenient to define, for $a \geq 0$,

$$
\phi_{n}(a)=\left(\int_{0}^{a} e^{-t^{2} / 2} t^{n-1} d t\right)^{1 / n}
$$


Then if $C$ is a Borel star set in $\mathbb{R}^{n}, n \geq 2$, a change to polar coordinates yields

$$
\gamma_{n}(C)=c_{n} \int_{S^{n-1}} \int_{0}^{\rho_{C}(u)} e^{-r^{2} / 2} r^{n-1} d r d u=c_{n} \int_{S^{n-1}} \phi_{n}\left(\rho_{C}(u)\right)^{n} d u,
$$

where $c_{n}$ is given by (9), an analog of the familiar polar coordinate expression for the $V_{n}$-measure of a Borel star set.

If $C$ is a Borel set contained in the ball $\varepsilon B$ for $\varepsilon>0$, it follows from (8) that

$$
c_{n} e^{-\varepsilon^{2} / 2} V_{n}(C) \leq \gamma_{n}(C) \leq c_{n} V_{n}(C) .
$$

\section{Coefficients of Dilatation in KNOWn IneQualities}

Since $\gamma_{n}$ is not homogeneous, it makes sense to carefully examine the precise conditions on the coefficients of dilatation in inequalities involving Gauss measure.

In [7] (see also [2]), Borell resolved this issue for Ehrhard's inequality (5) by showing that

$$
\Phi^{-1}\left(\gamma_{n}(s K+t L)\right) \geq s \Phi^{-1}\left(\gamma_{n}(K)\right)+t \Phi^{-1}\left(\gamma_{n}(L)\right),
$$

where $\Phi$ is defined by (12), holds for $s, t \geq 0$, even for Borel sets, when $s+t \geq 1$ and $|s-t| \leq 1$, and not generally unless these conditions are satisfied. In [8], Borell shows that, remarkably, the corresponding condition for convex $K$ and $L$ is different; here only $s+t \geq 1$ is required.

The corresponding analysis for the weaker inequality (6) does not appear in the literature as far as we know. We claim that the inequality

$$
\gamma_{n}(s K+t L) \geq \gamma_{n}(K)^{s} \gamma_{n}(L)^{t}
$$

holds generally for Borel star sets $K$ and $L$ and $s, t \geq 0$ if and only if $s+t \geq 1$. To see this, note first that if $s+t<1$ and $K=L$, (16) implies that

$$
\gamma_{n}(K)>\gamma_{n}((s+t) K) \geq \gamma_{n}(K)^{s+t},
$$

a contradiction since $\gamma_{n}(K) \leq 1$. Suppose, then, that $s+t \geq 1$. Let

$$
f(s, t)=\log \left(\gamma_{n}(s K+t L)\right)-s \log \left(\gamma_{n}(K)\right)-t \log \left(\gamma_{n}(L)\right) .
$$

Clearly $\gamma_{n}(s K+t L)$ increases with $s$ and $t, \log \left(\gamma_{n}(K)\right) \leq 0$, and $\log \left(\gamma_{n}(L)\right) \leq 0$, so $\partial f / \partial s \geq 0$ and $\partial f / \partial t \geq 0$. If $s, t \geq 1$, this yields $f(s, t) \geq f(0,1)=0$, as required. On the other hand if $t<1$, say, then $f(s, t) \geq f(1-t, t) \geq 0$ by (6), completing the proof of the claim.

Note, however, that for convex $K$ and $L$, (16) holds generally for $s, t \geq 0$ if and only if $s=1-t$. In view of the previous paragraph, we need only consider the case when $s+t>1$. Let $n=1$, let $K=L=[x, x+1], x>0$, and let $s+t=a>1$. Then (16) and crude estimates give

or

$$
\frac{a}{\sqrt{2 \pi}} e^{-(a x)^{2} / 2}>\gamma_{1}([a x, a x+a]) \geq \gamma_{1}([x, x+1])^{a}>\left(\frac{1}{\sqrt{2 \pi}} e^{-(x+1)^{2} / 2}\right)^{a}
$$

$$
\frac{a}{\sqrt{2 \pi}} e^{-a^{2} x^{2} / 2}>\frac{1}{(2 \pi)^{a / 2}} e^{-a(x+1)^{2} / 2} .
$$

Since $a^{2}>a$, this is clearly false for sufficiently large $x$.

In view of the connection (15) between Gauss and Lebesgue measure, we revisit the classical and dual Brunn-Minkowski inequality for exponents $p>0$.

To deal with this, first note that if $p>0$ and $a, b, s, t \geq 0$, the weighted pth means $\left(s a^{p}+t b^{p}\right)^{1 / p}$ increase with $p$ for all $a, b \geq 0$ if and only if $s+t \leq 1$ and 
decrease with $p$ for all $a, b \geq 0$ if and only if $s, t \geq 1$. (The cases $s=1-t$ and $s=t=1$ are usually called the pth mean and pth sum of $a$ and $b$, respectively.) See [20, (2.10.4) and (2.10.5), p. 29]. In particular, the inequality

$$
(s a+t b)^{p} \geq s a^{p}+t b^{p}
$$

is true for all $a, b \geq 0$ when $p=1$, when $p<1$ and $s+t \leq 1$, and when $p>1$ and $s, t \geq 1$, and it is false for all $a, b>0$ when $p>1$ and $s+t \leq 1$, and when $p<1$ and $s, t \geq 1$. Moreover, it does not generally hold otherwise. To see this, it suffices to check that when $s<1$ and $t>1$, (17) is false for $p<1$ and sufficiently small $a$ and for $p>1$ and sufficiently small $b$.

The above monotonicity properties of the weighted means and (2) imply that

$$
V_{n}(s K+t L)^{p} \geq s V_{n}(K)^{p}+t V_{n}(L)^{p}
$$

holds for $s, t \geq 0$ and all convex bodies $K$ and $L$ in $\mathbb{R}^{n}$ when $p=1 / n$, when $0<p<1 / n$ and $s+t \leq 1$, and when $p>1 / n$ and $s, t \geq 1$. By using the homogeneity of volume and the remarks above concerning the inequality (17), we see that (18) is otherwise generally false for $K=a B, L=b B$ and small $a, b \geq 0$, and it is always false for $K=a B, L=b B, a, b>0$, when $p>1 / n$ and $s+t \leq 1$, and when $0<p<1 / n$ and $s, t \geq 1$.

In a similar fashion, it can be seen that

$$
V_{n}(s C \widetilde{+} t D)^{p} \leq s V_{n}(C)^{p}+t V_{n}(D)^{p}
$$

holds for $s, t \geq 0$ and all bounded Borel star sets $C$ and $D$ in $\mathbb{R}^{n}$ when $p=1 / n$, when $p>1 / n$ and $s+t \leq 1$, and when $0<p<1 / n$ and $s, t \geq 1$. It is otherwise generally false for $C=a B, D=b B$ and small $a, b \geq 0$, and it is always false for $C=a B, D=b B, a, b>0$, when $0<p<1 / n$ and $s+t \leq 1$, and when $p>1 / n$ and $s, t \geq 1$.

\section{A Gaussian dual Brunn-Minkowski inequality}

Lemma 4.1. The function $\phi_{n}$ defined by (13) is sublinear, i.e.,

$$
\phi_{n}(a+b) \leq \phi_{n}(a)+\phi_{n}(b)
$$

for $a, b \geq 0$, with equality if and only if $a=0$ or $b=0$.

Proof. For fixed $b>0$ and all $a \geq 0$, define

$$
f(a)=\phi_{n}(a+b)-\phi_{n}(a)-\phi_{n}(b) .
$$

Then $f(0)=0$, and it suffices to show that $f^{\prime}(a)<0$ for all $a \geq 0$. In view of (13), we have

$$
n f^{\prime}(a)=(a+b)^{n-1} e^{-(a+b)^{2} / 2} \phi_{n}(a+b)^{1-n}-a^{n-1} e^{-a^{2} / 2} \phi_{n}(a)^{1-n} .
$$

If $n=1$, it is clear from this that $f^{\prime}(a)<0$ for $a \geq 0$. Suppose that $n \geq 2$. Using (13) again, we see that $f^{\prime}(a)<0$ is equivalent to

$$
(a+b)^{-n} e^{n(a+b)^{2} /(2(n-1))} \int_{0}^{a+b} e^{-t^{2} / 2} t^{n-1} d t>a^{-n} e^{n a^{2} /(2(n-1))} \int_{0}^{a} e^{-t^{2} / 2} t^{n-1} d t
$$

or

$$
e^{n(a+b)^{2} /(2(n-1))} \int_{0}^{1} e^{-(s(a+b))^{2} / 2} s^{n-1} d s>e^{n a^{2} /(2(n-1))} \int_{0}^{1} e^{-(s a)^{2} / 2} s^{n-1} d s .
$$


Rearranging, we obtain

$$
\int_{0}^{1} e^{\left(n /(n-1)-s^{2}\right)(a+b)^{2} / 2} s^{n-1} d s>\int_{0}^{1} e^{\left(n /(n-1)-s^{2}\right) a^{2} / 2} s^{n-1} d s .
$$

The previous inequality holds since $s^{2} \leq 1<n /(n-1)$, and this proves the lemma.

Theorem 4.2. Let $C$ and $D$ be Borel star sets in $\mathbb{R}^{n}$, and let $s, t \geq 1$. Then

$$
\gamma_{n}(s C \tilde{+} t D)^{1 / n} \leq s \gamma_{n}(C)^{1 / n}+t \gamma_{n}(D)^{1 / n} .
$$

Suppose that $C$ and $D$ are properly contained in $\mathbb{R}^{n}$. Equality holds when $s=t=1$ if and only if $\gamma_{n}(C)=0, \gamma_{n}(D)=0$, or $n=1$ and both $C$ and $D$ are (possibly degenerate or infinite) intervals with one endpoint at the origin, each on opposite sides of the origin. Equality holds when $s>1$ and $t=1$ (or $s=1$ and $t>1$, or $s>1$ and $t>1$ ) if and only if $\gamma_{n}(C)=0$ (or if and only if $\gamma_{n}(D)=0$, or if and only if $\gamma_{n}(C)=0$ and $\gamma_{n}(D)=0$, respectively).

Proof. Suppose first that $s=t=1$.

If $n=1$ and $C$ and $D$ are bounded, then $C=\left[-a_{1}, b_{1}\right]$ and $D=\left[-a_{2}, b_{2}\right]$ for nonnegative $a_{1}, a_{2}, b_{1}$, and $b_{2}$, and (20) is equivalent to

$$
\phi_{1}\left(a_{1}+a_{2}\right)+\phi_{1}\left(b_{1}+b_{2}\right) \leq\left(\phi_{1}\left(a_{1}\right)+\phi_{1}\left(b_{1}\right)\right)+\left(\phi_{1}\left(a_{2}\right)+\phi_{1}\left(b_{2}\right)\right) .
$$

This follows immediately from Lemma 4.1, and its equality condition shows that either $a_{1}=0$ or $a_{2}=0$ and either $b_{1}=0$ or $b_{2}=0$. The same conclusion is reached if $C$ or $D$ is unbounded. This yields the required equality condition when $n=1$.

Suppose that $n \geq 2$. By (14), (7), Lemma 4.1, and Minkowski's inequality for integrals, we have

$$
\begin{aligned}
\gamma_{n}(C \tilde{+} D)^{1 / n} & =\left(c_{n} \int_{S^{n-1}} \phi_{n}\left(\rho_{C \tilde{+} D}(u)\right)^{n} d u\right)^{1 / n} \\
& =\left(c_{n} \int_{S^{n-1}} \phi_{n}\left(\rho_{C}(u)+\rho_{D}(u)\right)^{n} d u\right)^{1 / n} \\
& \leq\left(c_{n} \int_{S^{n-1}}\left(\phi_{n}\left(\rho_{C}(u)\right)+\phi_{n}\left(\rho_{D}(u)\right)\right)^{n} d u\right)^{1 / n} \\
& \leq\left(c_{n} \int_{S^{n-1}} \phi_{n}\left(\rho_{C}(u)\right)^{n} d u\right)^{1 / n}+\left(c_{n} \int_{S^{n-1}} \phi_{n}\left(\rho_{D}(u)\right)^{n} d u\right)^{1 / n} \\
& =\gamma_{n}(C)^{1 / n}+\gamma_{n}(D)^{1 / n} .
\end{aligned}
$$

Suppose, in addition to our assumption that $s=t=1$, that equality holds in (20). Then for almost all $u \in S^{n-1}$, equality holds in Lemma 4.1 when $a=\rho_{C}(u)$ and $b=\rho_{D}(u)$, and hence for almost all $u \in S^{n-1}$ we have either $\rho_{C}(u)=0$ or $\rho_{D}(u)=0$. But equality also holds in Minkowski's inequality for integrals, so there is a constant $c$ such that $\phi_{n}\left(\rho_{C}(u)\right)=c \phi_{n}\left(\rho_{D}(u)\right)$ for almost all $u \in S^{n-1}$. It follows that either $\rho_{C}(u)=0$ for almost all $u \in S^{n-1}$ or $\rho_{D}(u)=0$ for almost all $u \in S^{n-1}$, and therefore either $\gamma_{n}(C)=0$ or $\gamma_{n}(D)=0$. 
We have proved (20) and its equality conditions when $s=t=1$. Using this and (11), for general $s, t \geq 1$ we obtain

$$
\gamma_{n}(s C \widetilde{+} t D)^{1 / n} \leq \gamma_{n}(s C)^{1 / n}+\gamma_{n}(t D)^{1 / n} \leq s \gamma_{n}(C)^{1 / n}+t \gamma_{n}(D)^{1 / n},
$$

as required. The equality conditions for $s>1$ or $t>1$ follow from those of (11).

Inequality (20) does not hold generally when either $s<1$ or $t<1$. Indeed, if $s<1$, (20) is false when $D=\varepsilon B$ and $\varepsilon>0$ is sufficiently small, in view of (11).

Inequality (20) is false for arbitrary Borel sets star shaped at the origin. To see this, let $s=t=1$, and for each $m \in \mathbb{N}$, let $C_{m}=\left\{(r, \theta) \in \mathbb{R}^{2}: m \leq r \leq m+1,0 \leq\right.$ $\theta \leq \pi / 2\}$ and $D_{m}=-C_{m}$. Then $C_{m} \widetilde{+} D_{m}=C_{0} \cup\left(-C_{0}\right)$, so $\gamma_{2}\left(C_{m} \widetilde{+} D_{m}\right)$ is positive and independent of $m$ while $\gamma_{2}\left(C_{m}\right)=\gamma_{2}\left(D_{m}\right) \rightarrow 0$ as $m \rightarrow \infty$. Note that $C_{m}$ and $D_{m}$ are actually star bodies.

The monotonicity properties of the weighted $p$ th means $\left(s a^{p}+t b^{p}\right)^{1 / p}$ summarized at the end of Section 2 imply that Theorem 4.2 holds for $s, t \geq 1$ and $0<p \leq 1 / n$. However, the exponent $1 / n$ in (20) is the best possible; it does not hold when $1 / n$ is replaced by $p>1 / n$, as can be seen by taking $C=a B$ and $D=b B$ for sufficiently small positive $a$ and $b$, and using (15) and the remarks concerning (19). Similarly, using the remarks concerning (18) instead, we see that it is also not true that (20) holds when $1 / n$ is replaced by $p>1 / n$ and the inequality is reversed.

When $C$ and $D$ are convex bodies containing the origin, we have $s C \widetilde{+} t D \subset$ $s C+t D$, so in this case the inequality $\gamma_{n}(s C+t D)^{1 / n} \leq s \gamma_{n}(C)^{1 / n}+t \gamma_{n}(D)^{1 / n}$ would be stronger than (20). However, by (2), its equality condition, and (15), this is false in general when $C$ and $D$ are sufficiently small nonhomothetic convex bodies containing the origin.

As a final remark in this section, we consider the possibility that

$$
\Theta_{n}^{-1}\left(\gamma_{n}(s C \tilde{+} t D)\right) \leq s \Theta_{n}^{-1}\left(\gamma_{n}(C)\right)+t \Theta_{n}^{-1}\left(\gamma_{n}(D)\right)
$$

holds for Borel star sets $C$ and $D$ in $\mathbb{R}^{n}$ and $s, t \geq 1$, where $\Theta_{n}$ is some standard function related to Gauss measure. Certainly (21) is not generally true when $s=$ $t=1$ and $\Theta_{n}=\Psi_{n}$, the function defined by (10). To see this, let $C$ and $D$ be half-spaces in $\mathbb{R}^{n}$ bounded by a common hyperplane through the origin, so that $C \tilde{+} D=\mathbb{R}^{n}$ and $\gamma_{n}(C)=\gamma_{n}(D)=1 / 2$. Then the left-hand side of (21) with $s=t=1$ and $\Theta_{n}=\Psi_{n}$ is infinite, while the right-hand side is bounded. Of course the same argument shows that (21) is not generally true when $\Theta_{n}=\Psi_{1}$ or $\Theta_{n}=\Phi$ (defined by (12)).

\section{Gaussian Brunn-Minkowski inequalities for o-Symmetric Balls}

In view of Theorem 4.2 and the dual Brunn-Minkowski inequality in the form (19), it is natural to ask whether there is a $p>0$ such that

$$
\gamma_{n}(s C \tilde{+} t D)^{p} \leq s \gamma_{n}(C)^{p}+t \gamma_{n}(D)^{p}
$$

holds for $s, t \geq 0, s+t \leq 1$, and Borel star sets $C$ and $D$ in $\mathbb{R}^{n}$. We shall see that the answer is negative for $s, t>0$, even for $o$-symmetric balls. To this end, the following lemma will be useful.

Lemma 5.1. The function

$$
F_{n}(r)=\left(\int_{0}^{r} e^{-t^{2} / 2} t^{n-1} d t\right)^{p}
$$


is strictly concave when (i) $0<p<1$ and $r \geq \sqrt{n-1}$, (ii) $p \geq 1$ and $r>\sqrt{n p-1}$, and (iii) $0<p \leq 1 / n$ and $r>0$.

Proof. Let

$$
I_{n}(r)=\int_{0}^{r} e^{-t^{2} / 2} t^{n-1} d t,
$$

so that $F_{n}(r)=I_{n}(r)^{p}$. A straightforward calculation yields

$$
F_{n}^{\prime \prime}(r)=p I_{n}(r)^{p-2} e^{-r^{2} / 2} r^{n-2}\left((p-1) e^{-r^{2} / 2} r^{n}+I_{n}(r)\left(n-1-r^{2}\right)\right) .
$$

Note that a trivial estimate gives $I_{n}(r)>e^{-r^{2} / 2} r^{n} / n$ for $r>0$, so if $r \geq \sqrt{n-1}$, we obtain $F_{n}^{\prime \prime}(r) \leq p I_{n}(r)^{p-2} e^{-r^{2}} r^{2 n-2}\left(n p-1-r^{2}\right) / n$. From this we see that $F_{n}^{\prime \prime}(r)<0$ when, in addition, $p<1$, establishing (i), and (ii) also follows immediately.

In proving (iii) we may suppose that $p=1 / n$, since $p$ th means increase with $p$. Substituting $p=1 / n$ into (25), we see that it suffices to show that

$$
G_{n}(r)=-(n-1) e^{-r^{2} / 2} r^{n}+n I_{n}(r)\left(n-1-r^{2}\right)<0
$$

for $r>0$. Now $G_{n}(0)=0$, and

$$
G_{n}^{\prime}(r)=-e^{-r^{2} / 2} r^{n+1}-2 n r I(r)<0
$$

for $r>0$. It follows that $G_{n}(r)<0$ for $r>0$, as required.

No attempt was made to obtain best possible estimates in cases (i) and (ii) of the previous lemma, since those found are sufficient for our purposes. Case (iii) of the previous lemma is equivalent to the concavity of $\phi_{n}(r)$ for $r>0$, and this is also implied by a result of Koenig and Tomczak-Jaegermann [21, p. 1218].

Corollary 5.2. Let $s, t \geq 0, s+t \leq 1$, and let $C$ and $D$ be o-symmetric balls in $\mathbb{R}^{n}$. Then

$$
\gamma_{n}(s C \tilde{+} t D)^{p} \geq s \gamma_{n}(C)^{p}+t \gamma_{n}(D)^{p}
$$

holds for $0<p \leq 1 / n$. Equality holds for $s, t>0$ if and only if $C=D$.

Proof. Note that when $n=1, \gamma_{1}(r B)=\gamma_{1}([-r, r])=2 c_{1} I_{1}(r)$, where $I_{n}(r)$ is given by (24). If $n \geq 2$, by (14), we have

$$
\gamma_{n}(r B)=c_{n} \int_{S^{n-1}} \phi_{n}(r)^{n} d u=n \kappa_{n} c_{n} I_{n}(r)
$$

for $r>0$. Thus if the function $F_{n}(r)$ given by (23) is concave for $0<a<r<b$, then

$$
\gamma_{n}((1-t) C \tilde{+} t D)^{p} \geq(1-t) \gamma_{n}(C)^{p}+t \gamma_{n}(D)^{p}
$$

holds when $C=r_{0} B, D=r_{1} B$, and $0<a<r_{0}, r_{1}<b$. By Lemma 5.1 (iii), $F_{n}(r)$ is actually strictly concave for $0<p \leq 1 / n$, and this yields the corollary together with the equality condition when $s=1-t$.

For general $s, t \geq 0$ with $s+t \leq 1$, let $\alpha=s /(1-t) \leq 1$ and note that by (27) and (11), for $0<p \leq 1 / n$, we have

$$
\begin{aligned}
\gamma_{n}(s C \tilde{+} t D)^{p}=\gamma_{n}((1-t)(\alpha C) \widetilde{+} t D)^{p} & \geq(1-t) \gamma_{n}(\alpha C)^{p}+t \gamma_{n}(D)^{p} \\
& \geq(1-t) \alpha^{p n} \gamma_{n}(C)^{p}+t \gamma_{n}(D)^{p} \\
& \geq(1-t) \alpha \gamma_{n}(C)^{p}+t \gamma_{n}(D)^{p} \\
& =s \gamma_{n}(C)^{p}+t \gamma_{n}(D)^{p}
\end{aligned}
$$


as required. If equality holds, then equality holds in (11), implying that $\alpha=1$, and then $C=D$ from the equality condition for (27).

Corollary 5.3. For given $s, t>0, s+t \leq 1$, and $p>0$, inequality (22) is false in general, even for o-symmetric balls.

Proof. Corollary 5.2 and its equality condition yield the result for $0<p \leq 1 / n$.

Suppose that $p>1 / n$. By Lemma 5.1(i) and (ii) we can choose the radii of $o$-symmetric balls $C$ and $D$ in $\mathbb{R}^{n}$ so that with $s=1-t$,

$$
\gamma_{n}(s C \widetilde{+} t D)^{p}>s \gamma_{n}(C)^{p}+t \gamma_{n}(D)^{p},
$$

and therefore so that (22) is false. It remains to consider the case when $s+t<1$. Let $C=a B$ and $D=a B$ for $a>0$. Then (28) is equivalent to

$$
\gamma_{n}((s+t) a B)^{p}>(s+t) \gamma_{n}(a B)^{p} .
$$

As $a \rightarrow \infty$, the left-hand side approaches 1 , while the right-hand side approaches $s+t<1$. It follows that (28) holds for sufficiently large $a$.

Note that Corollary 5.2 holds even for $p<0$, at least when $s=1-t$. This is because $p$ th means increase with real $p$; see [20, Section 2.9]. Consequently Corollary 5.3 also holds when $s=1-t$ and $p<0$.

Corollary 5.2 does not hold in general, even when both $C$ and $D$ are dilatates of a fixed $o$-symmetric Borel star set $E$. To see this, let $E_{1}=\left\{(x, y) \in \mathbb{R}^{2}: x, y \geq 0\right\}$, $E_{2}(a)=\left\{(r, \theta) \in \mathbb{R}^{2}: 0 \leq r \leq a, \pi / 2 \leq \theta \leq \pi\right\}$, and let $E(a)=E_{1} \cup\left(-E_{1}\right) \cup$ $E_{2}(a) \cup\left(-E_{2}(a)\right)$. Letting

$$
f(t)=\gamma_{2}(t E(a))^{1 / 2}=\left(\frac{1}{2}+\frac{1}{2}\left(1-e^{-t^{2} a^{2} / 2}\right)\right)^{1 / 2}=I(t)^{1 / 2},
$$

say, we obtain

$$
f^{\prime \prime}(t)=\frac{a^{2} e^{-t^{2} a^{2} / 2}}{16 I(t)^{3 / 2}}\left(-t^{2} a^{2} e^{-t^{2} a^{2} / 2}+4 I(t)\left(1-t^{2} a^{2}\right)\right) .
$$

Using the inequalities $1-x \leq e^{-x} \leq 1-x+x^{2} / 2$ for $x \geq 0$, we have

$$
\begin{aligned}
-t^{2} a^{2} e^{-t^{2} a^{2} / 2}+4 I(t)\left(1-t^{2} a^{2}\right) & =4-4 t^{2} a^{2}-2 e^{-t^{2} a^{2} / 2}+t^{2} a^{2} e^{-t^{2} a^{2} / 2} \\
& \geq \frac{1}{4}\left(8-8 t^{2} a^{2}-3 t^{4} a^{4}\right)
\end{aligned}
$$

The latter quantity is positive for $0 \leq t \leq 1$, and hence $f(t)$ is convex there, when $a \leq a_{0}=((2 \sqrt{10}-4) / 3)^{1 / 2}=0.8802 \ldots$ It follows that if $0<a_{1}<a_{2}<a_{0}$, $C=E\left(a_{1}\right)$, and $D=E\left(a_{2}\right)$, then (26) is false for $0<s=1-t<1$ when $n=2$ and $p=1 / 2$. By replacing $E_{1}$ with $E_{1}^{\prime}=\left\{(r, \theta) \in \mathbb{R}^{2}: 0 \leq r \leq b, 0 \leq \theta \leq \pi / 2\right\}$ for sufficiently large $b$ and then approximating, we can clearly also find sets $C$ and $D$ in $\mathbb{R}^{2}$, each dilatates of a fixed $o$-symmetric star body, such that (26) is false for $0<s=1-t<1$ when $n=2$ and $p=1 / 2$.

\section{More on Gaussian dual Brunn-Minkowski inequalities}

The results of the previous section and the existence of Ehrhard's inequality (5) raise the following question. 
Question 6.1. Let $n \in \mathbb{N}$. Is there a natural nonconstant function $\Theta_{n}$ such that for $0<t<1$ and Borel star sets $C$ and $D$ in $\mathbb{R}^{n}$,

$$
\Theta_{n}^{-1}\left(\gamma_{n}((1-t) C \tilde{+} t D)\right) \leq(1-t) \Theta_{n}^{-1}\left(\gamma_{n}(C)\right)+t \Theta_{n}^{-1}\left(\gamma_{n}(D)\right) ?
$$

For $n=1$, we can take $\Theta_{1}=1-\Phi$, for then, noting that $1-\Phi(x)=\Phi(-x)$, we have $\Theta^{-1}=-\Phi^{-1}$, and since the radial sum equals the Minkowski sum when $n=1$, (29) becomes Ehrhard's inequality (5)! However, we cannot take $\Theta_{n}=1-\Phi$ when $n \geq 2$. To see this, note that this would imply that Ehrhard's inequality (5) is true when $n \geq 2, K$ and $L$ are Borel star sets, and the Minkowski sum is replaced by the radial sum. But this is false. Indeed, recall that since $\Phi$ is $\log$ concave, this would imply that (6) also holds when $n \geq 2, K$ and $L$ are Borel star sets, and the Minkowski sum is replaced by the radial sum. Moreover, from the equality conditions for (5) we can conclude that the radial sum version of (6) would hold with strict inequality when $K$ and $L$ are dilatates with $K \neq L$. By (15), we would then have

$$
V_{n}((1-t) K \widetilde{+} t L)>V_{n}(K)^{1-t} V_{n}(L)^{t}
$$

for sufficiently small nonequal dilatates $K$ and $L$. By a standard argument (see, for example, [14, p. 362]), this would contradict (4).

Any $\Theta_{n}$ for which (29) holds for $o$-symmetric Borel star sets must be decreasing. To see this, let $C$ and $D$ be $o$-symmetric infinite double cones such that $C \cap D=\{o\}$. Then $(1-t) C=C, t D=D$, and $(1-t) C \tilde{+} t D=C \cup D$. If $\gamma_{n}(C)=a$ and $\gamma_{n}(D)=b$, then (29) yields

$$
\Theta_{n}^{-1}(a+b) \leq(1-t) \Theta_{n}^{-1}(a)+t \Theta_{n}^{-1}(b) .
$$

As $t \rightarrow 0$, we obtain $\Theta_{n}^{-1}(a+b) \leq \Theta_{n}^{-1}(a)$. Therefore $\Theta_{n}^{-1}$ is decreasing on $[0,1]$ and hence $\Theta_{n}$ is also decreasing. In particular, we cannot take $\Theta_{n}=\Phi, \Psi_{1}$, or $\Psi_{n}$ (see (10) ).

Despite all this, we claim that for all $n \in \mathbb{N}$, (29) is true when $\Theta_{n}=\Psi_{1}, C=\{o\}$, and $D$ is $o$-symmetric and convex. To see this, let $0<t<1$ and consider an $o$ symmetric slab (the closed region between two parallel hyperplanes) $P$ of half-width $a$, and note that $\gamma_{n}(P)=\gamma_{1}([-a, a])=\Psi_{1}(a)$, or $a=\Psi_{1}^{-1}\left(\gamma_{n}(P)\right)$. Suppose that $P$ is chosen so that $\gamma_{n}(P)=\gamma_{n}(D)$. Then $P$ has half-width $\Psi_{1}^{-1}\left(\gamma_{n}(D)\right)$, so $t P$ has half-width $t \Psi_{1}^{-1}\left(\gamma_{n}(D)\right)$ and $\gamma_{n}(t P)=\Psi_{1}\left(t \Psi_{1}^{-1}\left(\gamma_{n}(D)\right)\right)$. By the so-called S-inequality (see [22] and 24]), we have

$$
\gamma_{n}(t D) \leq \gamma_{n}(t P)=\Psi_{1}\left(t \Psi_{1}^{-1}\left(\gamma_{n}(D)\right)\right),
$$

which is (29) for the special case under consideration.

The previous observation suggests that Question 6.1 should be revisited under the restriction that the sets $C$ and $D$ are $o$-symmetric closed convex sets. In fact, it turns out that we still cannot take $\Theta_{n}=\Phi, \Psi_{1}$, or $\Psi_{n}$, but different arguments are required.

To see that it is still not possible to take $\Theta_{n}=\Phi$, let $C$ and $D$ be different parallel $o$-symmetric slabs. Then $(1-t) C \widetilde{+} t D=(1-t) C+t D$, so (29) with $\Theta_{n}=\Phi$ would contradict (5) and its equality conditions.

Next, note that if Question 6.1 has a positive answer for o-symmetric closed convex sets, then $\Theta_{n}^{-1}\left(\Psi_{1}(x)\right)$ must be convex. Indeed, let $C$ and $D$ be parallel $o$-symmetric slabs of half-widths $x$ and $y$, respectively, so that $(1-t) C \tilde{+} t D$ is an $o$-symmetric slab of half-width $(1-t) x+t y$. Then $\gamma_{n}(C)=\Psi_{1}(x), \gamma_{n}(D)=\Psi_{1}(y)$, 
and $\gamma_{n}((1-t) C \widetilde{+} t D)=\Psi_{1}((1-t) x+t y)$, so (29) becomes

$$
\Theta_{n}^{-1}\left(\Psi_{1}((1-t) x+t y)\right) \leq(1-t) \Theta_{n}^{-1}\left(\Psi_{1}(x)\right)+t \Theta_{n}^{-1}\left(\Psi_{1}(y)\right)
$$

which holds for all $x, y \geq 0$ if and only if $\Theta_{n}^{-1}\left(\Psi_{1}(x)\right)$ is convex.

Let $f(x)=\Psi_{n}^{-1}\left(\Psi_{1}(x)\right), n \geq 2$. Using (14) and differentiating $\Psi_{n}(f(x))=\Psi_{1}(x)$ with respect to $x$, we obtain

$$
c_{n} n \kappa_{n} e^{-f(x)^{2} / 2} f(x)^{n-1} f^{\prime}(x)=\sqrt{\frac{2}{\pi}} e^{-x^{2} / 2}
$$

or

$$
f^{\prime}(x)=d_{n} \frac{e^{\left(f^{2}(x)-x^{2}\right) / 2}}{f(x)^{n-1}}
$$

for some constant $d_{n}$. It follows that

$$
f^{\prime \prime}(x)=-d_{n} \frac{e^{\left(f^{2}(x)-x^{2}\right) / 2}}{f(x)^{n}}\left(x f(x)+\left(n-1-f(x)^{2}\right) f^{\prime}(x)\right)
$$

As $x \rightarrow 0+$, we have $f(x) \rightarrow 0$ and $f^{\prime}(x) \rightarrow \infty$. Therefore $f^{\prime \prime}(x)$ must be negative for small $x$, so $f(x)$ is not convex. By the previous paragraph, we still cannot take $\Theta_{n}=\Psi_{n}$ for $n \geq 2$.

The previous argument does not eliminate the possibility $\Theta_{n}=\Psi_{1}$. To deal with this we first observe by taking $C$ and $D$ to be $o$-symmetric balls of radius $x$ and $y$, respectively, that if Question 6.1 has a positive answer for $o$-symmetric closed convex sets, then $\Theta_{n}^{-1}\left(\Psi_{n}(x)\right)$ must be convex. We shall show that $g(x)=$ $\Psi_{1}^{-1}\left(\Psi_{n}(x)\right)$ is not convex for $n=2$.

To this end, note first that $\Psi_{2}(x)=1-e^{-x^{2} / 2}, \Psi_{2}^{\prime}(x)=x e^{-x^{2} / 2}$, and $\Psi_{1}^{\prime}(x)=$ $\sqrt{2 / \pi} e^{-x^{2} / 2}$. By differentiating $\Psi_{1}(g(x))=\Psi_{2}(x)$, we obtain

$$
g^{\prime}(x)=\sqrt{\frac{\pi}{2}} x e^{\left(g^{2}-x^{2}\right) / 2}
$$

and hence

$$
g^{\prime \prime}(x)=\sqrt{\frac{\pi}{2}} e^{\left(g^{2}-x^{2}\right) / 2}\left(1+x\left(g^{\prime} g-x\right)\right) .
$$

So it suffices to study the sign of

$$
h(x)=1+x\left(g^{\prime} g-x\right)=1+x\left(\sqrt{\frac{\pi}{2}} x g e^{\left(g^{2}-x^{2}\right) / 2}-x\right) .
$$

From $\Psi_{1}(g(x))=\Psi_{2}(x)$ we also obtain

$$
\sqrt{\frac{2}{\pi}} \int_{0}^{g} e^{-t^{2} / 2} d t=1-e^{-x^{2} / 2}
$$


which yields

$$
\begin{aligned}
\sqrt{\frac{\pi}{2}} e^{-x^{2} / 2} & =\int_{g}^{\infty}(1 / t) t e^{-t^{2} / 2} d t \\
& =\frac{1}{g} e^{-g^{2} / 2}-\int_{g}^{\infty} \frac{1}{t^{2}} e^{-t^{2} / 2} d t \\
& =\frac{1}{g} e^{-g^{2} / 2}-\frac{1}{g^{3}} e^{-g^{2} / 2}+3 \int_{g}^{\infty} \frac{1}{t^{4}} e^{-t^{2} / 2} d t \\
& <\frac{1}{g} e^{-g^{2} / 2}-\frac{1}{g^{3}} e^{-g^{2} / 2}+3 \int_{g}^{\infty} \frac{t}{g^{5}} e^{-t^{2} / 2} d t \\
& =e^{-g^{2} / 2}\left(\frac{1}{g}-\frac{1}{g^{3}}+\frac{3}{g^{5}}\right) .
\end{aligned}
$$

From (30) and (33), we have

$$
h(x)<\frac{1}{g^{2}}\left(g^{2}-x^{2}+\frac{3 x^{2}}{g^{2}}\right) .
$$

Now (31) gives

and hence

$$
\sqrt{\frac{\pi}{2}} e^{\left(g^{2}-x^{2}\right) / 2}<\frac{1}{g}
$$

$$
g^{2}-x^{2}<-\ln \left(\pi g^{2} / 2\right) .
$$

Similarly (32) yields

$$
g^{2}-x^{2}>-\ln \left(\frac{\pi g^{6}}{2\left(g^{2}-1\right)^{2}}\right) .
$$

By (34), (35), and (36), we conclude that

$$
h(x)<\frac{1}{g^{2}}\left(-\ln \left(\pi g^{2} / 2\right)+3+\frac{3}{g^{2}} \ln \left(\frac{\pi g^{6}}{2\left(g^{2}-1\right)^{2}}\right)\right),
$$

which is negative for sufficiently large $x$, since $g(x) \rightarrow \infty$ as $x \rightarrow \infty$.

\section{A possible Gaussian Brunn-Minkowski inequality}

Question 7.1. Let $0<t<1$ and let $K$ and $L$ be closed convex sets containing the origin in $\mathbb{R}^{n}$. Is it true that

$$
\gamma_{n}((1-t) K+t L)^{1 / n} \geq(1-t) \gamma_{n}(K)^{1 / n}+t \gamma_{n}(L)^{1 / n} ?
$$

The exponent $1 / n$ is the best possible. Indeed, by the relation (15) and the remarks after (18) concerning the classical Brunn-Minkowski inequality with exponent $p$, we see that (37) does not hold in general when $1 / n$ is replaced by $p>1 / n$. On the other hand, if (37) is true, then the remarks in Section 2 about the weighted $p$ th means ensure that (37) remains true when $1 / n$ is replaced by $0<p \leq 1 / n$.

A positive answer to Question 7.1 would imply that (37) remains true when $(1-t)$ is replaced by $s>0$, under the condition $s+t \leq 1$, as can be verified by the same argument used at the end of the proof of Corollary 5.2.

We gave an example after Corollary $[5.3$ showing that the stronger inequality

$$
\gamma_{n}((1-t) K \widetilde{+} t L)^{1 / n} \geq(1-t) \gamma_{n}(K)^{1 / n}+t \gamma_{n}(L)^{1 / n}
$$


is false in general for $K$ and $L$ which are both dilatates of the same $o$-symmetric star body. It is also false for sufficiently small star bodies $K$ and $L$ containing the origin that are not dilatates, by (4), its equality condition, and (15).

Some restriction on the position of the sets is necessary. To see this, let $0<t<1$, $K=B$, and $L=B+x_{1} e_{1}$, where $x_{1}>0$ and $e_{1}$ is a unit vector in the direction of the positive first coordinate axis. Then $(1-t) K+t L=B+t x_{1} e_{1}$, so the left-hand side of (37) approaches zero as $x_{1} \rightarrow \infty$, while the right-hand side remains bounded away from zero.

If it is true, (37) would be stronger than (6) for closed convex sets containing the origin, and it does not follow from Ehrhard's inequality (5). Indeed, we claim that this is even the case when $K$ and $L$ are $o$-symmetric balls. To prove this, for fixed $0<t<1$ and $r_{0}>0$, consider the function

$$
\begin{aligned}
f(r)= & \Phi\left((1-t) \Phi^{-1}\left(\gamma_{n}\left(r_{0} B\right)\right)+t \Phi^{-1}\left(\gamma_{n}(r B)\right)\right) \\
& -\left((1-t) \gamma_{n}\left(r_{0} B\right)^{1 / n}+t \gamma_{n}(r B)^{1 / n}\right)^{n} .
\end{aligned}
$$

If $r_{0}$ is chosen so that $\gamma_{n}\left(r_{0} B\right)=1 / 2$, then $\Phi^{-1}\left(\gamma_{n}\left(r_{0} B\right)\right)=0$ and we have $f(r)<0$ if and only if

$$
\Phi\left(t \Phi^{-1}\left(\gamma_{n}(r B)\right)\right)<\left((1-t) 2^{-1 / n}+t \gamma_{n}(r B)^{1 / n}\right)^{n}
$$

or

$$
t \Phi^{-1}\left(\gamma_{n}(r B)\right)<\Phi^{-1}\left(\left((1-t) 2^{-1 / n}+t \gamma_{n}(r B)^{1 / n}\right)^{n}\right) .
$$

Now as $r \rightarrow 0+$, the left-hand side of the previous inequality approaches $-\infty$, while the right-hand approaches $\Phi^{-1}\left((1-t)^{n} / 2\right)$. Therefore $f(r)<0$ for sufficiently small $r>0$, proving the claim.

Corollary 5.2 shows that the answer to Question 7.1 is positive if $K$ and $L$ are $o$-symmetric balls, since in this case the radial sum and Minkowski sum coincide.

Theorem 7.2. Question 7.1 has a positive answer when $n=1$.

Proof. Let $0<t<1$ and let $K=[-a, b]$ and $L=[-c, d]$ for nonnegative reals $a, b$, $c$, and $d$. Note that since $n=1$, radial and Minkowski addition coincide. Then, by the first statement of Corollary 5.2 with $n=1$, we have

$$
\begin{aligned}
\gamma_{1}((1-t) K+t L)= & \gamma_{1}((1-t)[-a, b]+t[-c, d]) \\
= & \gamma_{1}([-(1-t) a-t c, 0])+\gamma_{1}([0,(1-t) b+t d]) \\
= & \frac{1}{2} \gamma_{1}((1-t)[-a, a]+t[-c, c]) \\
& \quad+\frac{1}{2} \gamma_{1}((1-t)[-b, b]+t[-d, d]) \\
\geq & \frac{1}{2}\left((1-t) \gamma_{1}([-a, a])+t \gamma_{1}([-c, c])\right) \\
& \quad+\frac{1}{2}\left((1-t) \gamma_{1}([-b, b])+t \gamma_{1}([-d, d])\right) \\
= & (1-t) \gamma_{1}([-a, 0])+t \gamma_{1}([-c, 0]) \\
& +(1-t) \gamma_{1}([0, b])+t \gamma_{1}([0, d]) \\
= & (1-t) \gamma_{1}([-a, b])+t \gamma_{1}([-c, d]) \\
= & (1-t) \gamma_{1}(K)+t \gamma_{1}(L),
\end{aligned}
$$


as required. The argument still applies if one or both of $K$ and $L$ is an infinite interval.

The following theorem generalizes the previous result. A different generalization is given in Theorem 8.2 .

Theorem 7.3. Question 7.1 has a positive answer when $K$ and $L$ are coordinate boxes containing the origin in $\mathbb{R}^{n}$.

Proof. Let $0<t<1$, and let $K=\prod_{i=1}^{n} I_{i}$ and $L=\prod_{i=1}^{n} J_{i}$ for closed (possibly unbounded) intervals $I_{i}$ and $J_{i}$ in $\mathbb{R}$ containing the origin, $1 \leq i \leq n$. Then

$$
(1-t) K+t L=\prod_{i=1}^{n}\left((1-t) I_{i}+t J_{i}\right) .
$$

An inequality of Minkowski (see [20, (2.13.8), p. 35]) states that for nonnegative reals $x_{i}$ and $y_{i}, 1 \leq i \leq n$,

$$
\left(\prod_{i=1}^{n}\left(x_{i}+y_{i}\right)\right)^{1 / n} \geq\left(\prod_{i=1}^{n} x_{i}\right)^{1 / n}+\left(\prod_{i=1}^{n} y_{i}\right)^{1 / n} .
$$

Using the fact that Gauss measure is a product measure, Theorem 7.2 , and (38), we obtain

$$
\begin{aligned}
\gamma_{n}((1-t) K+t L)^{1 / n} & =\left(\prod_{i=1}^{n} \gamma_{1}\left((1-t) I_{i}+t J_{i}\right)\right)^{1 / n} \\
& \geq\left(\prod_{i=1}^{n}\left((1-t) \gamma_{1}\left(I_{i}\right)+t \gamma_{1}\left(J_{i}\right)\right)\right)^{1 / n} \\
& \geq\left(\prod_{i=1}^{n}\left((1-t) \gamma_{1}\left(I_{i}\right)\right)\right)^{1 / n}+\left(\prod_{i=1}^{n}\left(t \gamma_{1}\left(J_{i}\right)\right)\right)^{1 / n} \\
& =(1-t) \gamma_{n}(K)^{1 / n}+t \gamma_{n}(L)^{1 / n}
\end{aligned}
$$

Corollary 7.4. Question 7.1 has a positive answer when one set is a slab containing the origin in $\mathbb{R}^{n}$.

Proof. Without loss of generality, let $L=[-a, b] \times \mathbb{R}^{n-1}, a, b \geq 0$, be a slab, and let $K_{S}=[-c, d] \times \mathbb{R}^{n-1}, c, d \geq 0$, be a parallel slab such that the hyperplanes $x_{1}=-c$ and $x_{1}=d$ support $K$. Then $K \subset K_{S}$ and $(1-t) K+t L=(1-t) K_{S}+t L$. Therefore, by Theorem 7.3 .

$$
\begin{aligned}
\gamma_{n}((1-t) K+t L)^{1 / n} & =\gamma_{n}\left((1-t) K_{S}+t L\right)^{1 / n} \\
& \geq(1-t) \gamma_{n}\left(K_{S}\right)^{1 / n}+t \gamma_{n}(L)^{1 / n} \\
& \geq(1-t) \gamma_{n}(K)^{1 / n}+t \gamma_{n}(L)^{1 / n} .
\end{aligned}
$$

Our next result is related to the so-called (B) conjecture proposed by W. Banaszczyk, which asks whether the function $\gamma_{n}\left(e^{t} K\right)$ is log concave in $t$ when $K$ is an $o$-symmetric closed convex set in $\mathbb{R}^{n}$. This was proved by Cordero-Erasquin, Fradelizi, and Maurey [10]. The following lemma merely rephrases the log concavity and is essentially part of the proof in [10] (see inequality (4) in that paper). 
Lemma 7.5. Let $K$ be a closed convex set in $\mathbb{R}^{n}$ such that $\gamma_{n}(K)>0$. Then $\gamma_{n}\left(e^{t} K\right)$ is $\log$ concave in $t$ if and only if

$$
\frac{\int_{K}\|x\|^{4} e^{-\|x\|^{2} / 2} d x}{\gamma_{n}(K)}-\left(\frac{\int_{K}\|x\|^{2} e^{-\|x\|^{2} / 2} d x}{\gamma_{n}(K)}\right)^{2}-2 \frac{\int_{K}\|x\|^{2} e^{-\|x\|^{2} / 2} d x}{\gamma_{n}(K)} \leq 0
$$

Theorem 7.6. Let $K_{0}$ be a closed convex set containing the origin in $\mathbb{R}^{n}$ such that $\gamma_{n}\left(K_{0}\right)>0$, and suppose that $\gamma_{n}\left(e^{t} K_{0}\right)$ is log concave in $t$. Then Question 7.1 has a positive answer when $K$ and $L$ are both dilatates of $K_{0}$.

Proof. Let $K_{0}$ satisfy the hypotheses of the theorem and define

$$
f(t)=c_{n}^{-1 / n} \gamma_{n}\left(t K_{0}\right)^{1 / n} .
$$

For $m=0,1,2, \ldots$, let

$$
I_{K_{0}, m}(t)=\int_{K_{0}}\|x\|^{m} e^{-t^{2}\|x\|^{2} / 2} d x=t^{-(m+n)} \int_{t K_{0}}\|x\|^{m} e^{-\|x\|^{2} / 2} d x=t^{-(m+n)} I_{L, m}(1),
$$

where $L=t K_{0}$. Then

$$
f(t)=\left(\int_{t K_{0}} e^{-\|x\|^{2} / 2} d x\right)^{1 / n}=t I_{K_{0}, 0}(t)^{1 / n} .
$$

Note that

$$
I_{K_{0}, m}^{\prime}(t)=-t I_{K_{0}, m+2}(t) .
$$

To prove the theorem, it suffices to show that $f(t)$ is concave for $0<t<1$. By direct calculation, using (40), we find

$$
f^{\prime}(t)=\frac{I_{K_{0}, 0}(t)^{1 / n}}{n}\left(n-t^{2} \frac{I_{K_{0}, 2}(t)}{I_{K_{0}, 0}(t)}\right)
$$

and

$$
\begin{aligned}
f^{\prime \prime}(t) & =\frac{t I_{K_{0}, 0}(t)^{1 / n}}{n^{2}}\left(t^{2}\left(n \frac{I_{K_{0}, 4}(t)}{I_{K_{0}, 0}(t)}-(n-1)\left(\frac{I_{K_{0}, 2}(t)}{I_{K_{0}, 0}(t)}\right)^{2}\right)-3 n \frac{I_{K_{0}, 2}(t)}{I_{K_{0}, 0}(t)}\right) \\
& =\frac{I_{L, 0}(1)^{1 / n}}{n^{2} t^{2}}\left(n \frac{I_{L, 4}(1)}{I_{L, 0}(1)}-(n-1)\left(\frac{I_{L, 2}(1)}{I_{L, 0}(1)}\right)^{2}-3 n \frac{I_{L, 2}(1)}{I_{L, 0}(1)}\right) \\
(41) & =\frac{I_{L, 0}(1)^{1 / n}}{n^{2} t^{2}}\left(n J_{L}+\left(\frac{I_{L, 2}(1)}{I_{L, 0}(1)^{2}}\right)\left(I_{L, 2}(1)-n I_{L, 0}(1)\right)\right),
\end{aligned}
$$

where

$$
J_{L}=\frac{I_{L, 4}(1)}{I_{L, 0}(1)}-\left(\frac{I_{L, 2}(1)}{I_{L, 0}(1)}\right)^{2}-2 \frac{I_{L, 2}(1)}{I_{L, 0}(1)}
$$


Now

$$
\begin{aligned}
I_{L, 2}(1) & =\int_{L}\|x\|^{2} e^{-\|x\|^{2} / 2} d x \\
& =\int_{S^{n-1}} \int_{0}^{\rho_{L}(u)} e^{-r^{2} / 2} r^{n+1} d r d u \\
& =-\int_{S^{n-1}} \rho_{L}(u)^{n} e^{-\rho_{L}(u)^{2} / 2} d u+n \int_{S^{n-1}} \int_{0}^{\rho_{L}(u)} e^{-r^{2} / 2} r^{n-1} d r d u \\
& \leq n \int_{S^{n-1}} \int_{0}^{\rho_{L}(u)} e^{-r^{2} / 2} r^{n-1} d r d u=n I_{L, 0}(1) .
\end{aligned}
$$

By (41) and (42), it suffices to show that $J_{L} \leq 0$. But this is precisely (39) with $K$ replaced by $L=t K_{0}$. Our assumption that $g(t)=\log \gamma_{n}\left(e^{t} K_{0}\right)$ concave in $t$ implies that for any $s>0, g(t+\log s)=\log \gamma_{n}\left(e^{t}\left(s K_{0}\right)\right)$ is concave, so (39) also holds when $K$ is replaced by any dilatate of $K_{0}$. This completes the proof.

As was mentioned above, the (B) conjecture was proved by Cordero-Erasquin, Fradelizi, and Maurey [10]. The same authors state that they do not know if the $o$-symmetry is needed, and they show that in some cases it is not. Specifically, they define $G(K)$ to be the group of isometries $\phi$ of $\mathbb{R}^{n}$ such that $\phi K=K$, and they define

$$
\operatorname{Fix}(K)=\left\{x \in \mathbb{R}^{n}: \phi x=x \text { for all } \phi \in G(K)\right\} .
$$

Then, in [10, Section 3], it is proved that $\gamma_{n}\left(e^{t} K\right)$ is $\log$ concave in $t$ when $\operatorname{Fix}(K)=$ $\{o\}$; for example, when $K$ is a regular simplex with centroid at the origin.

Corollary 7.7. Question 7.1 has a positive answer when $K$ and $L$ are both dilatates of the same o-symmetric closed convex set, or more generally, of the same closed convex set $K_{0}$ with $\operatorname{Fix}\left(K_{0}\right)=\{o\}$.

We remark that calculations very similar to those in the example given just before Question 6.1 show that the function $\gamma_{n}\left(e^{t} K\right)$ is not log concave in general when $K$ is an $o$-symmetric star body. Indeed, let $E_{1}=\left\{(x, y) \in \mathbb{R}^{2}: x, y \geq 0\right\}, E_{2}(a)=$ $\left\{(r, \theta) \in \mathbb{R}^{2}: 0 \leq r \leq a, \pi / 2 \leq \theta \leq \pi\right\}$, and $E(a)=E_{1} \cup\left(-E_{1}\right) \cup E_{2}(a) \cup\left(-E_{2}(a)\right)$. Define

$$
f(t)=\log \left(\gamma_{2}\left(e^{t} E(a)\right)\right)=\log \left(\frac{1}{2}+\frac{1}{2}\left(1-e^{-e^{2 t} a^{2} / 2}\right)\right)=\log I(t),
$$

say. Then

$$
f^{\prime \prime}(t)=\frac{e^{2 t} a^{2} e^{-e^{2 t} a^{2} / 2}}{2 I(t)^{2}}\left(2-e^{2 t} a^{2}-e^{-e^{2 t} a^{2} / 2}\right) .
$$

Using the inequality $e^{-x} \leq 1-x+x^{2} / 2$ for $x=e^{2 t} a^{2} / 2 \geq 0$, we have

$$
2-e^{2 t} a^{2}-e^{-e^{2 t} a^{2} / 2} \geq \frac{1}{8}\left(8-4 e^{2 t} a^{2}-e^{4 t} a^{4}\right) .
$$

The latter quantity is positive for $0 \leq t \leq 1$, and hence $f(t)$ is convex there, when

$$
a \leq a_{0}=(2 \sqrt{3}-2)^{1 / 2} e^{-1}=0.4451 \ldots
$$

If we replace $E_{1}$ with $E_{1}^{\prime}=\left\{(r, \theta) \in \mathbb{R}^{2}: 0 \leq r \leq b, 0 \leq \theta \leq \pi / 2\right\}$ for sufficiently large $b$ and approximate, we can find an $o$-symmetric star body $E$ such that $\gamma_{n}\left(e^{t} E\right)$ is not $\log$ concave. 
For some time we considered the possibility that if $0<t<1$ and $K$ and $L$ are $o$-symmetric closed convex sets in $\mathbb{R}^{n}$, then

$$
\Psi_{n}^{-1}\left(\gamma_{n}((1-t) K+t L)\right) \geq(1-t) \Psi_{n}^{-1}\left(\gamma_{n}(K)\right)+t \Psi_{n}^{-1}\left(\gamma_{n}(L)\right),
$$

where $\Psi_{n}$ is defined by (10), with equality if and only if $K$ and $L$ are $o$-symmetric balls. The motivation was the fact that for arbitrary convex sets $K$ and $L$, (43) implies (37). Indeed, using (43) and the fact that by the first statement of Corollary 5.2 the function $\Psi_{n}(r)^{1 / n}$ is concave for $r>0$, we obtain

$$
\begin{aligned}
\gamma_{n}((1-t) K+t L)^{1 / n} & \geq \Psi_{n}\left((1-t) \Psi_{n}^{-1}\left(\gamma_{n}(K)\right)+t \Psi_{n}^{-1}\left(\gamma_{n}(L)\right)\right)^{1 / n} \\
& \geq(1-t) \Psi_{n}\left(\Psi_{n}^{-1}\left(\gamma_{n}(K)\right)\right)^{1 / n}+t \Psi_{n}\left(\Psi_{n}^{-1}\left(\gamma_{n}(L)\right)\right)^{1 / n} \\
& =(1-t) \gamma_{n}(K)^{1 / n}+t \gamma_{n}(L)^{1 / n},
\end{aligned}
$$

which is (37).

However, inequality (43) is false in general for arbitrary o-symmetric convex sets. We are grateful to Franck Barthe for the following proof of this fact. (A similar argument is used by Latała [22, p. 816].)

Let $K$ and $L$ be $o$-symmetric convex sets in $\mathbb{R}^{n}$, let $0<t<1$, and let $h>0$. In (43), replace $K$ by $(1-t)^{-1} K$ and let $L=(h / t) B$. Then, on letting $t \rightarrow 0$, we obtain from (43) the inequality

$$
\Psi_{n}^{-1}\left(\gamma_{n}(K+h B)\right) \geq \Psi_{n}^{-1}\left(\gamma_{n}(K)\right)+h .
$$

Choose $r>0$ so that $\gamma_{n}(r B)=\gamma_{n}(K)$. Then (44) yields

$$
\gamma_{n}(K+h B) \geq \Psi_{n}\left(\Psi_{n}^{-1}\left(\gamma_{n}(r B)\right)+h\right)=\Psi_{n}(r+h)=\gamma_{n}(r B+h B) .
$$

Therefore

$$
\lim _{h \rightarrow 0+} \frac{\gamma_{n}(K+h B)-\gamma_{n}(K)}{h} \geq \lim _{h \rightarrow 0+} \frac{\gamma_{n}(r B+h B)-\gamma_{n}(r B)}{h} .
$$

However, by [25, Lemma 3], the previous inequality is false when $n=2, K=$ $\left\{(x, y) \in \mathbb{R}^{2}: y \in[-a, a]\right\}$ is a slab, and $a>0$ is sufficiently large.

Indeed, it can be seen by direct calculation that (43) is false when $K=\{(x, y) \in$ $\left.\mathbb{R}^{2}: y \in[-1 /(1-t), 1 /(1-t)]\right\}, L=(1 / t) B$, and $0<t<0.04$. It is interesting to note that by Corollary 7.4, sets of this form cannot supply a negative answer to Question 7.1.

\section{A Gaussian PréKopa-Leindler inequality}

If $f$ is a nonnegative measurable function on $\mathbb{R}^{n}$ and $s \geq 0$, the superlevel set $L(f, s)$ is defined by

$$
L(f, s)=\{x: f(x) \geq s\} .
$$

Note that

$$
\begin{aligned}
c_{n} \int_{\mathbb{R}^{n}} f(x) e^{-\|x\|^{2} / 2} d x & =c_{n} \int_{\mathbb{R}^{n}} \int_{0}^{f(x)} e^{-\|x\|^{2} / 2} d s d x \\
& =c_{n} \int_{0}^{\infty} \int_{L(f, s)} e^{-\|x\|^{2} / 2} d x d s=\int_{0}^{\infty} \gamma_{n}(L(f, s)) d s .
\end{aligned}
$$

The standard Prékopa-Leindler inequality (see, for example, [14, Theorem 7.1]) holds when Lebesgue measure is replaced by any log concave measure, in particular, 
by $\gamma_{n}$. Theorem 7.2 yields the following stronger inequality when $n=1$, for a restricted class of functions.

Theorem 8.1. Let $0<t<1$ and let $f, g$, and $h$ be nonnegative integrable functions on $\mathbb{R}$ such that superlevel sets of $f$ and $g$ are either empty or intervals containing the origin. If

$$
h((1-t) x+t y) \geq(1-t) f(x)+t g(y)
$$

for all $x, y \in \mathbb{R}$, then

$$
\int_{\mathbb{R}} h(x) e^{-\|x\|^{2} / 2} d x \geq(1-t) \int_{\mathbb{R}} f(x) e^{-\|x\|^{2} / 2} d x+t \int_{\mathbb{R}} g(x) e^{-\|x\|^{2} / 2} d x .
$$

Proof. If $s \geq 0, f(x) \geq s$, and $g(y) \geq s$, then $h((1-t) x+t y) \geq s$. Therefore,

$$
L(h, s) \supseteq(1-t) L(f, s)+t L(g, s) .
$$

Then, by (45), the fact that $L(f, s)$ and $L(g, s)$ are intervals containing the origin, and Theorem 7.2 , we obtain

$$
\begin{aligned}
\int_{\mathbb{R}} h(x) e^{-\|x\|^{2} / 2} d x & =\frac{1}{c_{1}} \int_{0}^{\infty} \gamma_{1}(L(h, s)) d s \\
& \geq \frac{1}{c_{1}} \int_{0}^{\infty} \gamma_{1}((1-t) L(f, s)+t L(g, s)) d s \\
& \geq \frac{1-t}{c_{1}} \int_{0}^{\infty} \gamma_{1}(L(f, s)) d s+\frac{t}{c_{1}} \int_{0}^{\infty} \gamma_{1}(L(g, s)) d s \\
& =(1-t) \int_{\mathbb{R}} f(x) e^{-\|x\|^{2} / 2} d x+t \int_{\mathbb{R}} g(x) e^{-\|x\|^{2} / 2} d x
\end{aligned}
$$

We do not know whether the assumption on the superlevel sets of $f$ and $g$ is necessary. It could be removed if Theorem 7.2 holds when $K$ and $L$ are arbitrary Borel sets containing the origin. In this direction, we have the following generalization of Theorem 7.2, inspired by work of Latała 23].

Theorem 8.2. Question 7.1 has a positive answer when $n=1, K$ is an interval containing the origin, and $L$ is any Borel set containing the origin.

Proof. Let $K=[a, b]$ and $L=\bigcup_{i=-m}^{n}\left[x_{i}, y_{i}\right]$, where

$$
x_{-m} \leq y_{-m}<x_{-m-1} \leq y_{-m-1}<\cdots<x_{n} \leq y_{n},
$$

$o \in[a, b]$, and $o \in\left[x_{0}, y_{0}\right]$. Then

$$
(1-t) K+t L=\bigcup_{i=-m}^{n}\left[(1-t) a+t x_{i},(1-t) b+t y_{i}\right] .
$$

We claim that we may assume that the intervals in this union are disjoint. Otherwise, for some $-m \leq i \leq n$, since $x_{i}<x_{i+1}$ and $y_{i}<y_{i+1}$, we have

$$
\begin{aligned}
\emptyset & \neq\left[(1-t) a+t x_{i},(1-t) b+t y_{i}\right] \cap\left[(1-t) a+t x_{i+1},(1-t) b+t y_{i+1}\right] \\
& =\left[(1-t) a+t x_{i},(1-t) b+t y_{i+1}\right]=(1-t)[a, b]+t\left[x_{i}, y_{i+1}\right] .
\end{aligned}
$$

Let

$$
L^{\prime}=\bigcup_{k=-m, k \neq i, i+1}^{n}\left[x_{k}, y_{k}\right] \cup\left[x_{i}, y_{i+1}\right] \text {. }
$$


Then $(1-t) K+t L=(1-t) K+t L^{\prime}$ and $\gamma_{1}\left(L^{\prime}\right) \geq \gamma_{1}(L)$, the set $L^{\prime}$ consists of fewer intervals than $L$, and $o \in L^{\prime}$. So we may replace $L$ by $L^{\prime}$. We can repeat the argument, if necessary, until all the intervals in the union are disjoint.

Since $o \in[a, b]$, we have

$$
\bigcup_{i=-m}^{n}\left[(1-t) a+t x_{i},(1-t) b+t y_{i}\right] \supseteq\left[(1-t) a+t x_{0},(1-t) b+t y_{0}\right] \cup \bigcup_{i=-m, i \neq 0}^{n}\left[t x_{i}, t y_{i}\right] \text {. }
$$

Now we can use Theorem 7.2 and (11) to obtain

$$
\begin{aligned}
\gamma_{1}((1-t) K+t L) & \geq \gamma_{1}\left(\left[(1-t) a+t x_{0},(1-t) b+t y_{0}\right] \cup \bigcup_{i=-m, i \neq 0}^{n}\left[t x_{i}, t y_{i}\right]\right) \\
& =\gamma_{1}\left(\left[(1-t) a+t x_{0},(1-t) b+t y_{0}\right]\right)+\sum_{i=-m, i \neq 0}^{n} \gamma_{1}\left(t\left[x_{i}, y_{i}\right]\right) \\
& \geq(1-t) \gamma_{1}(K)+t \gamma_{1}\left(\left[x_{0}, y_{0}\right]\right)+t \sum_{i=-m, i \neq 0}^{n} \gamma_{1}\left(\left[x_{i}, y_{i}\right]\right) \\
& =(1-t) \gamma_{1}(K)+t \gamma_{1}(L) .
\end{aligned}
$$

Therefore the result holds when $L$ is a finite union of intervals, and the theorem is then proved by a standard approximation argument.

Of course, the previous result allows the assumptions in Theorem 8.1 to be weakened.

\section{REFERENCES}

[1] F. Barthe, The Brunn-Minkowski theorem and related geometric and functional inequalities, in: International Congress of Mathematicians, Vol. II, Eur. Math. Soc., Zürich, 2006, pp. 1529-1546. MR2275657 (2007k:39047)

[2] F. Barthe and N. Huet, On Gaussian Brunn-Minkowski inequalities, Studia Math. 191 (2009), 283-304. MR2481898 (2010f:60052)

[3] C. Borell, Convex measures on locally convex spaces, Ark. Math. 12 (1974), 239-252. MR0388475 (52:9311)

[4] C. Borell, Convex set functions in $d$-space, Period. Math. Hungar. 6 (1975), 111-136. MR0404559 (53:8359)

[5] C. Borell, The Brunn-Minkowski inequality in Gauss space, Invent. Math. 30 (1975), 207-216. MR0399402(53:3246)

[6] C. Borell, The Ehrhard inequality, C. R. Math. Acad. Sci. Paris 337 (2003), 663-666. MR2030108 (2004k:60102)

[7] C. Borell, Minkowski sums and Brownian exit times, Ann. Fac. Sci. Toulouse Math. 16 (2007), 37-47. MR2325590

[8] C. Borell, Inequalities of the Brunn-Minkowski type for Gaussian measures, Probab. Theory Relat. Fields 140 (2008), 195-205. MR.2357675

[9] H. J. Brascamp and E. Lieb, Some inequalities for Gaussian measures and the long-range order of one-dimensional plasma, in Functional Integration and Its Applications, ed. by A. M. Arthurs, Clarendon Press, Oxford, 1975, pp. 1-14.

[10] D. Cordero-Erausquin, M. Fradelizi, and B. Maurey, The (B) conjecture for the Gaussian measure of dilates of symmetric convex sets and related problems, J. Funct. Anal. 214 (2004), 410-427. MR2083308 (2005g:60064)

[11] A. Ehrhard, Symétrisation dans l'espace de Gauss, Math. Scand. 53 (1983), 281-301. MR745081 (85f:60058)

[12] A. Ehrhard, Élements extrémaux pours les inégalités de Brunn-Minkowski gaussienes, Ann. Inst. H. Poincaré Probab. Statist. 22 (1986), 149-168. MR850753 (88a:60041) 
[13] R. J. Gardner, Geometric Tomography, second edition, Cambridge University Press, New York, 2006. MR2251886 (2007i:52010)

[14] R. J. Gardner, The Brunn-Minkowski inequality, Bull. Amer. Math. Soc. 39 (2002), 355-405. MR:1898210 (2003f:26035)

[15] R. J. Gardner, Intersection bodies and the Busemann-Petty problem, Trans. Amer. Math. Soc. 342 (1994), 435-445. MR1201126 (94e:52008)

[16] R. J. Gardner, A positive answer to the Busemann-Petty problem in three dimensions, Ann. of Math. (2) 140 (1994), 435-447. MR1298719 (95i:52005)

[17] R. J. Gardner, A. Koldobsky, and T. Schlumprecht, An analytical solution to the BusemannPetty problem on sections of convex bodies, Ann. of Math. (2) 149 (1999), 691-703. MR1689343 (2001b:52011)

[18] R. J. Gardner, E. B. Vedel Jensen, and A. Volčič, Geometric tomography and local stereology, Adv. in Appl. Math. 30 (2003), 397-423. MR1973951(2004e:28006)

[19] R. J. Gardner and A. Volčič, Tomography of convex and star bodies, Adv. Math. 108 (1994), 367-399. MR.1296519 (95j:52013)

[20] G. H. Hardy, J. E. Littlewood, and G. Pólya, Inequalities, Cambridge University Press, Cambridge, 1959.

[21] H. Koenig and N. Tomczak-Jaegermann, Geometric inequalities for a class of exponential measures, Proc. Amer. Math. Soc. 133 (2004), 1213-1221. MR2117224 (2005m:46022)

[22] R. Latała, On some inequalities for Gaussian measures, in Proc. Internat. Congress of Mathematicians, Vol. II (Beijing, 2002), Higher Ed. Press, Beijing, 2002, pp. 813-822. MR.1957087 (2004b:60055)

[23] R. Latała, A note on the Ehrhard inequality, Studia Math. 118 (1996), 169-174. MR1389763 (97d:60027)

[24] R. Latała and K. Oleszkiewicz, Gaussian measures and dilatations of convex symmetric sets, Ann. Probab. 273 (1999), 1922-1938. MR.1742894 (2000k:60062)

[25] R. Latała and K. Oleszkiewicz, Small ball probability estimates in terms of widths, Studia Math. 169 (2005), 305-314. MR2140804 (2006f:60040)

[26] E. Lutwak, Intersection bodies and dual mixed volumes, Adv. Math. 71 (1988), 232-261. MR963487 (90a:52023)

[27] E. Lutwak, Dual mixed volumes, Pacific J. Math. 58 (1975), 531-538. MR0380631 (52:1528)

[28] B. Maurey, Inégalité de Brunn-Minkowski-Lusternik, et autres inégalités géométriques et fonctionnelles, in: Séminaire Bourbaki, Vol. 2003/2004, Astérisque No. 299 (2005), Exp. No. 928, vii, pp. 95-113. MR2167203 (2006g:52006)

[29] R. Schneider, Convex Bodies: The Brunn-Minkowski Theory, Cambridge University Press, Cambridge, 1993. MR1216521 (94d:52007)

[30] G. Zhang, Intersection bodies and the Busemann-Petty inequalities in $\mathbb{R}^{4}$, Ann. of Math. (2) 140 (1994), 331-346. MR1298716 (95i:52004)

[31] G. Zhang, A positive answer to the Busemann-Petty problem in four dimensions, Ann. of Math. (2) 149 (1999), 535-543. MR1689339(2001b:52010)

Department of Mathematics, Western Washington University, Bellingham, WashingTON 98225-9063

E-mail address: Richard.Gardner@wwu.edu

Department of Mathematics, Kent State University, Kent, Ohio 44242

E-mail address: zvavitch@math.kent.edu 